\title{
Trends in HIV Testing among Adults in Georgia: Analysis of the 2011-2015 BRFSS Data
}

\author{
Benjamin E. Ansa ${ }^{1, *}$, Sashia White ${ }^{2}$, Yunmi Chung ${ }^{1}$ and Selina A. Smith ${ }^{1,3}$ \\ 1 Institute of Public \& Preventive Health, Augusta University, CJ-2300 1120 15th Street, Augusta, GA 30912, \\ USA; ychung@augusta.edu (Y.C.); sesmith@augusta.edu (S.A.S.) \\ 2 Medical College of Georgia, Augusta University, 1120 15th Street, Augusta, GA 30912, USA; \\ saswhite@augusta.edu \\ 3 Department of Family Medicine, Medical College of Georgia, Augusta University, Augusta, GA 30912, USA \\ * Correspondence: bansa@augusta.edu; Tel.: +1-706-721-6141
}

Academic Editors: Ronald L. Braithwaite and Mario De La Rosa

Received: 2 September 2016; Accepted: 8 November 2016; Published: 11 November 2016

\begin{abstract}
Georgia is ranked fifth highest among states for rates of human immunodeficiency virus (HIV) diagnosis. About $4 \%$ of persons living with HIV infection in the United States reside in Georgia, and almost $19 \%$ of these people do not know their HIV status. The present study examined the trends and associated factors of HIV testing among adults in Georgia between 2011 and 2015 by analyzing data of the Behavioral Risk Factor Surveillance System (BRFSS). A total of 31,094 persons aged $\geq 18$ years were identified who responded to the question "Have you ever been tested for HIV?" Overall, there were 11,286 (44.2\%) respondents who had been tested for HIV, compared to 19,808 (55.8\%) who had not. There was a slight decrease in the percentage of respondents who have ever tested for HIV, from 45.6\% in 2011 to $43.7 \%$ in 2015 (APC (annual percent change $)=-0.98$, not significant). Factors associated with HIV testing were being female $(p=0.004)$, black ( $p<0.001)$, younger than 55 years $(p<0.001)$, single $(p<0.001)$, attaining education level above high school $(p<0.001)$, and earning annual income of $\$ 50,000$ or less $(p=0.028)$. Overall in Georgia, there has been a slight decline in the temporal trend of HIV testing, and more than half of adults have never been tested for HIV. For reducing HIV transmission in Georgia, enhancing access and utilization of HIV testing should be a public health priority.
\end{abstract}

Keywords: HIV/AIDS; testing; trends; Behavioral Risk Factor Surveillance System; sociodemographic; Georgia

\section{Introduction}

Human immunodeficiency virus (HIV) testing contributes to the prevention and control of HIV / AIDS. Following HIV diagnosis, risk behaviors tend to decrease [1-3]; people diagnosed with HIV can make decisions that potentially lower HIV transmission risk by avoiding risk behaviors such as unprotected sex and needle sharing [4]. Also, people who test negative for HIV can make decisions to protect themselves from HIV by engaging in safer sex behaviors and in some cases, taking pre-exposure prophylaxis [4]. Enabling individuals to become diagnosed early is a public health priority [5], as late diagnosis of HIV infection can lead to increased morbidity, mortality, and healthcare costs [5].

According to the Centers for Disease Control and Prevention (CDC), an estimated 1.2 million people in the United States (U.S.) are living with HIV, and 13\% $(156,300)$ of these do not know they are infected [6]. Each year, nearly 45,000 people are diagnosed with HIV, with $30 \%$ of new HIV infections being transmitted by people who are living with undiagnosed HIV [4]. Geographically, the burden of HIV is not evenly distributed. In 2014, the rates (per 100,000 people) of persons diagnosed with HIV 
infection were the highest in the South (18.5), followed by the Northeast (14.2), the West (11.2), and the Midwest (8.2) [6].

Georgia (GA) with a population of 10,214,860 in 2015, is the second most populous state in the southeast of the U.S. [7]. The population is made up of $62 \%$ whites, $32 \%$ blacks / African Americans, 9.0\% Hispanics/Latinos, and 5.0\% other races/ethnicities [7]. With a rate of 27 per 100,000, GA is ranked fifth highest among states in regard to rates of HIV diagnosis. According to the CDC, 1 in 51 Georgians will be diagnosed with HIV in their lifetime (compared to 1 in 670 residents of North Dakota) [8]. Despite comprising only $3.1 \%$ of the U.S. population in 2014, $6.4 \%$ (2640) of new HIV diagnoses and $4.4 \%(53,230)$ of persons living with HIV infection in the U.S. were recorded in GA [9]. About $19 \%$ of those living with HIV are unaware of their HIV status, and almost $23 \%$ of persons in the state of GA were diagnosed with AIDS within three months, as a result of late testing for HIV $[9,10]$. This means that they harbored the virus for a long period of time without receiving appropriate treatment that would have prevented further deterioration of their immune system.

In 2006, the CDC recommended that, as part of routine health care, everyone between the ages of 13 and 64 be tested for HIV at least once, with yearly HIV testing for high-risk individuals, in line with the National HIV / AIDS Strategy goal of increasing by 2015 the percentage (to $90 \%$ ) of persons living with HIV who know their serostatus [11,12]. The purpose of the present investigation was to evaluate the progress in HIV testing in GA by examining the temporal trends and factors associated with testing for HIV among adults residing in GA between 2011 and 2015.

\section{Materials and Methods}

\subsection{Study Design}

This cross-sectional study was done by analyzing nationally representative datasets.

\subsection{Data Source, Study Participants, and Sampling}

The Behavioral Risk Factor Surveillance System (BRFSS) is a nationally representative cross-sectional survey that collects data on U.S. residents in all 50 states, the District of Columbia, and three U.S. territories, regarding their health-related risk behaviors, chronic health conditions, and use of preventive services [13]. GA has been part of the system since it was established in 1984 [14]. Surveys are conducted through phone interviews (landline and cellphone) and more than 400,000 adult interviews are conducted each year, making it the largest continuously conducted health survey system in the world and a useful tool for addressing and developing health promotion activities [13].

Although conducted in different time periods, the surveys used identical methods for recruitment. GA is among the participating BRFSS states that utilize disproportionate stratified sample (DSS) design for their landline samples [15]. Telephone numbers are divided into two groups, or strata, which are sampled separately. The high-density and medium-density strata contain telephone numbers that are expected to belong mostly to households. Whether a telephone number goes into the high-density or medium-density stratum is determined by the number of listed household numbers in its hundred block, or set of 100 telephone numbers with the same area code, prefix, and first two digits of the suffix and all possible combinations of the last two digits. BRFSS puts numbers from hundred blocks with one or more listed household numbers (1+ blocks, or banks) in either the high-density stratum (listed $1+$ blocks) or medium-density stratum (unlisted $1+$ blocks). The BRFSS samples the two strata to obtain a probability sample of all households with telephones. Cellular telephone sampling frames are commercially available and the system can call random samples of cellular telephone numbers, but doing so requires specific protocols [15]. The basis of the 2011-2015 BRFSS sampling frame is the Telecordia database of telephone exchanges (e.g., 617-492-0000 to 617-492-9999) and 1000 banks (e.g., 617-492-0000 to 617-492-0999). The vendor uses dedicated cellular 1000 banks, sorted on the basis of area code and exchange within a state. The BRFSS forms an interval (K) by dividing the population count of telephone numbers in the frame $(\mathrm{N})$ by the desired sample size $(\mathrm{n})$. The BRFSS 
divides the frame of telephone numbers into $\mathrm{n}$ intervals of size $\mathrm{K}$ telephone numbers. From each interval, the BRFSS draws one 10-digit telephone number at random. In the sample design, each state begins with a single stratum. To provide adequate sample sizes for smaller geographically defined populations of interest, however, many states sample disproportionately from strata that correspond to substate regions.

Response rates for BRFSS were calculated using standards set by the American Association of Public Opinion Research (AAPOR) Response Rate Formula 4 [16]. The median survey response rate (\%) for all states and Washington, DC, in 2011 was 49.7, and ranged from 33.8 to 64.1 [17]; in 2012 was 45.2, and ranged from 27.7 to 60.4 [18]; in 2013 was 46.4, and ranged from 29.0 to 60.3 [19]; in 2014 was 47.0, and ranged from 25.1 to 60.1 [20]; and in 2015 was 47.2, and ranged from 33.9 to 61.1 [21]. Response rates (\%) for GA included in this analysis had a weighted AAPOR response rate of 49.9 in 2011 [17], 53.5 in 2012 [18], 46.5 in 2013 [19], 48.8 in 2014 [20], and 47.6 in 2015 [21].

Secondary analyses of the BRFSS 2011-2015 data were performed to identify persons in GA aged $\geq 18$ years who reported having ever been tested for HIV.

\subsection{Measures}

Respondents were categorized under socio-demographic variables of gender (male or female); age in years $(18-24,25-34,35-44,45-54,55-64$, or 65+); race (non-Hispanic (NH) white, NH black, Hispanic, NH other, or NH multiracial); education (<high school, high school/General Educational Development (GED), some post high school, or college graduate); annual income in United States Dollar (USD ( $<\$ 15,000, \$ 15,000-<\$ 25,000, \$ 25,000-<\$ 35,000, \$ 35,000-<\$ 50,000$, or $\$ 50,000+)$ ); marital status (married, divorced, widowed, separated, never married, or a member of an unmarried couple); healthcare coverage (yes/no); and HIV high-risk situations (yes/no). HIV high-risk situations included engaging in any of the following behaviors for the past year: use of intravenous drugs, treatment for sexually transmitted disease, giving or receiving money or drugs for sex, or having anal sex without a condom. The type of health coverage was only assessed for the year 2014 these data were available. The outcome variable was participants' response to the question "Have you ever been tested for HIV?" (yes/no).

\subsection{Statistical Analyses}

Descriptive statistics of socio-demographic variables and HIV high-risk situations related to HIV testing were generated for each year, using frequencies and proportions. Data were weighted using the iterative proportional fitting weighting method (i.e., raking) to adjust for noncoverage, nonresponse, and for differences between sample and population characteristics [22]. Weighted percentages of respondents who had ever been tested for HIV were calculated for each variable category for each year. Joinpoint Trend Analysis software [23,24] was used to calculate the annual percent change (APC) over time. The model is linear on the log of the response for calculating annual percentage rate change. An APC is computed for each of those trends by means of generalized linear models assuming a Poisson distribution. The tests of significance use a Monte Carlo Permutation method. Significant changes include changes in direction or in the rate of increase or decrease. Logistic regression analyses were conducted to examine the association between socio-demographic variables and HIV testing. The model included data for the five years under review (2011-2015), and data were adjusted for gender, age, race, education, income, marital status, and healthcare coverage. Some of the variables were merged and then compared with the reference category. For example, the variable marital status was re-categorized into single and couple; those that were divorced, widowed, separated and never married were grouped as single, and compared to those that were in a couple relationship (married, and a member of an unmarried couple). Similarly for age, the categories were collapsed into three (18-34-young adults; 35-54-middle age; and $\geq 55$ - older adults). The same was done for race, education, and income. Odds ratios and related $95 \%$ confidence intervals were derived from regression analysis. Pair-wise rate differences were examined using bivariate survey-weighted logistic regression. 
Chi-square test and Monte Carlo Permutation method were used to obtain $p$ values. The significance level was set at $p<0.05$, and all tests were two-sided. Unweighted counts, weighted percentages, and logistic regression analyses were performed using the IBM SPSS Complex Samples version 24 (IBM Corp., Armonk, NY, USA) [25].

\subsection{Ethical Considerations}

BRFSS datasets are publicly accessible and do not contain personally identifiable information. CDC ensures that the process of data collection and release are governed by appropriate rules, regulations, and legislative authorizations [26].

\section{Results}

\subsection{Socio-Demographic Characteristics and HIV Risk Situations of Respondents}

In the BRFSS database, between 2011 and 2015, 31,094 adults in GA responded to the question "Have you ever been tested for HIV?" The respondents were $\geq 18$ years old, predominantly female $(63.0 \%, n=19,545)$, white $(66.7 \%, n=20,743)$, college graduates $(34.9 \%, n=10,837)$, married $(51.4 \%$, $n=15,988)$, with an annual income of $\geq \$ 50,000(35.5 \%, n=11,032)$, and with some form of healthcare coverage $(86.0 \%, n=26,731)$ (Table 1$)$. In addition, for the years data were available (2011 and 2012), $97.6 \%(n=14,142)$ of respondents did not engage in HIV high-risk behaviors. For all the years under review, the results of the descriptive analyses of socio-demographic categories and having been tested for HIV were statistically significant $(p<0.001)$.

\subsection{Trends in HIV Testing among Adults in GA, 2011-2015}

In GA, there were 11,286 (44.2\%) respondents who had ever been tested for HIV. The weighted population estimates of those, by year of interview, and the APC for each variable are shown in Table 2. There was a slight decrease in the percentages of respondents who had ever been tested for HIV, from $45.6 \%$ in 2011 to $43.7 \%$ in 2015 (APC $=-0.98$, not significant). There was a significant decrease over time in the number of HIV testers among annual income earners of $<\$ 15,000$ (APC $=-2.29$ ). The percentages of HIV testers over time were stable and APCs were not significant among the other categories of socio-demographic variables.

Overall, for the period of 2011-2015 (results not shown in Table 2), approximately $60 \%$ of persons between the ages of 25 and 44 had been tested for HIV, compared to $<50 \%$ of those aged 18-24 and greater than 45 years of age $(p<0.001)$. Also, the highest percentages of testers were among NH black respondents $(62.4 \%$ vs. $35.2 \%$ white and $42.8 \%$ Hispanic $(p<0.001)$. Fewer persons with healthcare coverage compared with those with no coverage $(41.9 \%$ and $52.8 \%$, respectively, $p<0.001)$; and almost $50 \%$ of respondents with greater than high school education, earning annual income of $<\$ 15,000(50 \%)$, separated (67\%), or in an unmarried couple relationship (52\%) had been tested for HIV. More persons engaged in high-risk behaviors had been tested for HIV than those who had not (71.8\% vs. 43.7\%; $p<0.001)$. Data for HIV high-risk activities and for the type of healthcare coverage were available for only 2011-2012 and 2014, respectively. As shown in Figure 1, respondents on the military (Tricare) plan were the highest testers $(68.2 \%(95 \% \mathrm{CI}=58.9 \%-76.2 \%))$, followed by those on Medicaid $(66.9 \%$ $(95 \%$ CI $=56.1 \%-76.1 \%))$. 
Table 1. Socio-demographic characteristics and HIV risk situations of survey respondents to the question “Have you ever been tested for HIV?" in Georgia: 2011-2015 BRFSS data.

\begin{tabular}{|c|c|c|c|c|c|c|c|c|c|c|c|c|}
\hline & \multirow{2}{*}{$\begin{array}{c}\begin{array}{c}\text { Total } \\
(N=31,094)\end{array} \\
N\end{array}$} & \multicolumn{2}{|c|}{$\begin{array}{c}2011 \\
(n=8977)\end{array}$} & \multicolumn{2}{|c|}{$\begin{array}{c}2012 \\
(n=5554)\end{array}$} & \multicolumn{2}{|c|}{$\begin{array}{c}2013 \\
(n=7010)\end{array}$} & \multicolumn{2}{|c|}{$\begin{array}{c}2014 \\
(n=5551)\end{array}$} & \multicolumn{2}{|c|}{$\begin{array}{c}2015 \\
(n=4002)\end{array}$} & \multirow[t]{2}{*}{$p$-Value } \\
\hline & & $\mathbf{n}$ & $\%$ & $\mathbf{n}$ & $\%$ & $\mathbf{n}$ & $\%$ & $\mathbf{n}$ & $\%$ & $\mathbf{n}$ & $\%$ & \\
\hline Gender & & & & & & & & & & & & $<0.001$ \\
\hline Male & 11,549 & 3162 & 35.2 & 2062 & 37.1 & 2621 & 37.4 & 2132 & 38.4 & 1572 & 37.1 & \\
\hline Female & 19,545 & 5815 & 64.8 & 3492 & 62.9 & 4389 & 62.6 & 3419 & 61.6 & 2430 & 62.9 & \\
\hline Age (years) & & & & & & & & & & & & $<0.001$ \\
\hline $18-24$ & 1495 & 309 & 3.4 & 283 & 5.1 & 428 & 6.1 & 290 & 5.2 & 185 & 4.6 & \\
\hline $25-34$ & 2985 & 828 & 9.2 & 540 & 9.7 & 775 & 11.1 & 485 & 8.7 & 357 & 8.9 & \\
\hline $35-44$ & 3914 & 1170 & 13.0 & 697 & 12.6 & 943 & 13.4 & 658 & 11.9 & 446 & 11.2 & \\
\hline $45-54$ & 5605 & 1683 & 18.8 & 1037 & 18.7 & 1281 & 18.3 & 950 & 17.1 & 654 & 16.4 & \\
\hline $55-64$ & 7248 & 2177 & 24.3 & 1263 & 22.7 & 1630 & 23.2 & 1260 & 22.7 & 918 & 22.9 & \\
\hline $65+$ & 9847 & 2810 & 31.3 & 1734 & 31.2 & 1953 & 27.9 & 1908 & 34.4 & 1442 & 36.0 & \\
\hline Race & & & & & & & & & & & & $<0.001$ \\
\hline White, NH & 20,743 & 6192 & 69.0 & 3687 & 66.4 & 4504 & 64.2 & 3678 & 66.2 & 2682 & 69.0 & \\
\hline Black, NH & 7413 & 2012 & 22.4 & 1340 & 24.1 & 1766 & 25.2 & 1349 & 24.3 & 946 & 22.4 & \\
\hline Hispanic & 1212 & 341 & 3.8 & 206 & 3.7 & 308 & 4.4 & 217 & 3.9 & 140 & 3.8 & \\
\hline Other, NH & 850 & 216 & 2.4 & 187 & 3.4 & 210 & 3.0 & 120 & 2.2 & 117 & 2.4 & \\
\hline Multiracial, $\mathrm{NH}$ & 445 & 106 & 1.2 & 74 & 1.3 & 117 & 1.7 & 94 & 1.7 & 54 & 1.2 & \\
\hline Do not know/Refused & 431 & 110 & 1.2 & 60 & 1.1 & 105 & 1.5 & 93 & 1.7 & 63 & 1.2 & \\
\hline Education & & & & & & & & & & & & $<0.001$ \\
\hline$<$ High school & 3497 & 997 & 11.1 & 694 & 12.4 & 771 & 11.0 & 615 & 11.1 & 420 & 10.5 & \\
\hline High School/GED & 8593 & 2550 & 28.4 & 1605 & 28.9 & 1923 & 27.4 & 1469 & 26.5 & 1046 & 26.1 & \\
\hline Some Post High School & 8086 & 2314 & 25.8 & 1442 & 26.0 & 1825 & 26.1 & 1474 & 26.5 & 1031 & 25.8 & \\
\hline College Grad & 10,837 & 3090 & 34.4 & 1797 & 32.4 & 2477 & 35.3 & 1980 & 35.7 & 1493 & 37.3 & \\
\hline Do not know/Refused & 81 & 26 & 0.3 & 16 & 0.3 & 14 & 0.2 & 13 & 0.2 & 12 & 0.3 & \\
\hline
\end{tabular}


Table 1. Cont

\begin{tabular}{|c|c|c|c|c|c|c|c|c|c|c|c|c|}
\hline & \multirow{2}{*}{$\begin{array}{c}\begin{array}{c}\text { Total } \\
(N=31,094)\end{array} \\
\mathrm{N}\end{array}$} & \multicolumn{2}{|c|}{$\begin{array}{c}2011 \\
(n=8977)\end{array}$} & \multicolumn{2}{|c|}{$\begin{array}{c}2012 \\
(n=5554)\end{array}$} & \multicolumn{2}{|c|}{$\begin{array}{c}2013 \\
(n=7010)\end{array}$} & \multicolumn{2}{|c|}{$\begin{array}{c}2014 \\
(n=5551)\end{array}$} & \multicolumn{2}{|c|}{$\begin{array}{c}2015 \\
(n=4002)\end{array}$} & \multirow[t]{2}{*}{$p$-Value } \\
\hline & & $\mathbf{n}$ & $\%$ & $\mathbf{n}$ & $\%$ & $\mathbf{n}$ & $\%$ & $\mathbf{n}$ & $\%$ & $\mathbf{n}$ & $\%$ & \\
\hline Annual Income (USD (\$)) & & & & & & & & & & & & $<0.001$ \\
\hline$<15,000$ & 3511 & 995 & 11.1 & 697 & 12.6 & 825 & 11.8 & 610 & 11.0 & 384 & 9.6 & \\
\hline $15,000-<25,000$ & 5215 & 1536 & 17.1 & 996 & 17.9 & 1122 & 16.0 & 930 & 16.7 & 631 & 15.8 & \\
\hline $25,000-<35,000$ & 3260 & 998 & 11.1 & 595 & 10.7 & 738 & 10.5 & 564 & 10.2 & 365 & 9.1 & \\
\hline $35,000-<50,000$ & 3650 & 1079 & 12.0 & 625 & 11.3 & 856 & 12.2 & 640 & 11.5 & 450 & 11.2 & \\
\hline $50,000+$ & 11,032 & 3142 & 35.0 & 1917 & 34.5 & 2451 & 35.0 & 2043 & 36.8 & 1479 & 37.0 & \\
\hline Do not know/Refused & 4426 & 1227 & 13.7 & 724 & 13.0 & 1018 & 14.5 & 764 & 13.8 & 693 & 17.3 & \\
\hline Marital status & & & & & & & & & & & & $<0.001$ \\
\hline Married & 15,988 & 4835 & 53.9 & 2845 & 51.2 & 3461 & 49.4 & 2787 & 50.2 & 2060 & 51.5 & \\
\hline Divorced & 4571 & 1313 & 14.6 & 798 & 14.4 & 1073 & 15.3 & 808 & 14.5 & 579 & 14.5 & \\
\hline Widowed & 4482 & 1312 & 14.6 & 830 & 14.9 & 903 & 12.9 & 858 & 15.5 & 579 & 14.5 & \\
\hline Separated & 838 & 226 & 2.5 & 138 & 2.5 & 223 & 3.2 & 151 & 2.7 & 100 & 2.5 & \\
\hline Never married & 4430 & 1090 & 12.1 & 824 & 14.8 & 1142 & 16.3 & 809 & 14.6 & 565 & 14.1 & \\
\hline $\begin{array}{l}\text { A member of an } \\
\text { unmarried couple }\end{array}$ & 648 & 170 & 1.9 & 103 & 1.9 & 171 & 2.4 & 111 & 2.0 & 93 & 2.3 & \\
\hline Refused & 137 & 31 & 0.4 & 16 & 0.3 & 37 & 0.5 & 27 & 0.5 & 26 & 0.6 & \\
\hline Healthcare coverage & & & & & & & & & & & & $<0.001$ \\
\hline Yes & 26,731 & 7680 & 85.6 & 4695 & 84.5 & 5872 & 83.8 & 4866 & 87.6 & 3618 & 90.4 & \\
\hline No & 4269 & 1272 & 14.2 & 847 & 15.3 & 1116 & 15.9 & 664 & 12.0 & 370 & 9.2 & \\
\hline Do not know/Refused & 94 & 25 & 0.2 & 12 & 0.2 & 22 & 0.3 & 21 & 0.4 & 14 & 0.4 & \\
\hline HIV high risk situations & $N=14,497$ & $\begin{array}{c}n= \\
8959\end{array}$ & & $\begin{array}{c}n= \\
5538\end{array}$ & & & & & & & & $<0.001$ \\
\hline Yes & 309 & 185 & 2.1 & 124 & 2.2 & $\mathrm{~N} / \mathrm{A}$ & $\mathrm{N} / \mathrm{A}$ & $\mathrm{N} / \mathrm{A}$ & $\mathrm{N} / \mathrm{A}$ & & & \\
\hline No & 14,142 & 8748 & 97.6 & 5394 & 97.4 & $\mathrm{~N} / \mathrm{A}$ & $\mathrm{N} / \mathrm{A}$ & $\mathrm{N} / \mathrm{A}$ & $\mathrm{N} / \mathrm{A}$ & & & \\
\hline Do not know/Refused & 46 & 26 & 0.3 & 20 & 0.4 & $\mathrm{~N} / \mathrm{A}$ & $\mathrm{N} / \mathrm{A}$ & $\mathrm{N} / \mathrm{A}$ & $\mathrm{N} / \mathrm{A}$ & & & \\
\hline
\end{tabular}

Acronyms: NH—non-Hispanic, GED—General Educational Development, N/A—data not available, USD—United States Dollar. 
Table 2. Weighted percentages and annual percent change of adults who have ever been tested for HIV in Georgia by year of interview: 2011-2015 BRFSS data.

\begin{tabular}{|c|c|c|c|c|c|c|c|c|c|c|c|c|}
\hline & Total & & 2011 & & 2012 & & 2013 & & 2014 & & 2015 & \\
\hline & Unwt. N & Unwt. $\mathbf{N}$ & Wt. $\%(95 \% \mathrm{CI})$ & Unwt. N & Wt. $\%(95 \% \mathrm{CI})$ & Unwt. N & Wt. $\%(95 \%$ CI $)$ & Unwt. N & Wt. $\%(95 \% \mathrm{CI})$ & Unwt. N & Wt. $\%(95 \% \mathrm{CI})$ & APC * \\
\hline Overall GA & 11,286 & 3174 & $45.6(44.0,47.2)$ & 1961 & $44.3(42.4,46.1)$ & 2753 & $43.6(42.0,45.3)$ & 1990 & $43.7(41.9,45.5)$ & 1408 & $43.7(41.6,45.9)$ & -0.98 \\
\hline \multicolumn{13}{|l|}{ Gender } \\
\hline Male & 4376 & 1145 & $45.0(42.5,47.6)$ & 746 & $43.0(40.1,45.9)$ & 1101 & $43.6(41.1,46.2)$ & 802 & $41.9(39.2,44.7)$ & 582 & $43.3(40.0,46.5)$ & -1.02 \\
\hline Female & 6910 & 2029 & $46.1(44.1,48.0)$ & 1215 & $45.5(43.1,47.8)$ & 1652 & $43.6(41.6,45.6)$ & 1188 & $45.4(43.1,47.7)$ & 826 & $44.2(41.3,47.0)$ & -0.86 \\
\hline \multicolumn{13}{|l|}{ Age (years) } \\
\hline $18-24$ & 666 & 140 & $42.4(36.3,48.8)$ & 352 & $46.2(39.9,52.7)$ & 191 & $39.5(34.3,45.0)$ & 122 & $40.4(34.5,46.6)$ & 73 & $40.1(32.6,48.1)$ & -2.43 \\
\hline $25-34$ & 1902 & 540 & $65.4(61.1,69.6)$ & 352 & $62.5(57.2,67.4)$ & 493 & $62.6(58.2,66.9)$ & 297 & $60.9(55.8,65.8)$ & 220 & $62.9(56.9,68.6)$ & -1.03 \\
\hline $35-44$ & 2401 & 712 & $62.0(58.4,65.4)$ & 397 & $57.2(52.6,61.7)$ & 594 & $60.7(56.7,64.6)$ & 411 & $60.1(55.5,64.6)$ & 287 & $61.8(56.3,67.1)$ & 0.43 \\
\hline $45-54$ & 2656 & 769 & $46.0(43.0,49.0)$ & 494 & $49.3(45.4,53.2)$ & 615 & $44.4(40.9,47.9)$ & 461 & $46.8(42.9,50.7)$ & 317 & $47.9(43.3,52.5)$ & 0.29 \\
\hline $55-64$ & 2264 & 653 & $31.9(29.4,34.5)$ & 352 & $28.8(25.6,32.3)$ & 540 & $33.5(30.6,36.6)$ & 406 & $33.9(30.6,37.4)$ & 313 & $32.6(29.0,36.4)$ & 2.09 \\
\hline $65+$ & 1397 & 360 & $13.9(12.3,15.7)$ & 226 & $14.6(12.4,17.1)$ & 320 & $16.0(14.0,18.2)$ & 293 & $15.8(13.9,18.0)$ & 198 & $15.6(13.2,18.4)$ & 3.15 \\
\hline \multicolumn{13}{|l|}{ Race } \\
\hline White, $\mathrm{NH}$ & 6058 & 1716 & $36.9(35.1,38.8)$ & 983 & $33.4(31.2,35.6)$ & 1460 & $36.3(34.5,38.2)$ & 978 & $34.3(32.2,36.5)$ & 753 & $35.2(32.8,37.7)$ & -0.68 \\
\hline Black, NH & 4201 & 1127 & $65.3(62.1,68.4)$ & 753 & $65.6(62.1,68.9)$ & 970 & $59.3(56.0,62.6)$ & 772 & $63.4(59.9,66.8)$ & 490 & $58.3(53.9,62.8)$ & -2.58 \\
\hline Hispanic & 549 & 155 & $40.8(33.8,48.1)$ & 88 & $42.0(34.0,50.5)$ & 138 & $43.9(37.5,50.5)$ & 95 & $38.1(30.8,46.0)$ & 67 & $49.7(39.5,60.0)$ & 3.02 \\
\hline Multiracial, $\mathrm{NH}$ & 237 & 52 & $54.2(41.4,66.5)$ & 43 & $59.5(44.9,72.7)$ & 60 & $55.0(42.2,67.3)$ & 53 & $57.1(42.7,70.4)$ & 33 & $69.9(54.3,81.6)$ & 4.79 \\
\hline Other, NH & 326 & 79 & $39.1(30.6,48.3)$ & 75 & $40.2(31.4,49.6)$ & 82 & $29.8(20.3,39.3)$ & 49 & $39.7(28.4,51.0)$ & 41 & $37.1(26.9,48.5)$ & -1.17 \\
\hline $\begin{array}{c}\text { Do not } \\
\text { know/Refused }\end{array}$ & 172 & 45 & $53.6(39.8,66.8)$ & 19 & $39.3(22.5,59.0)$ & 43 & $45.5(34.0,57.6)$ & 43 & $53.6(39.9,66.8)$ & 24 & $43.2(26.8,61.3)$ & - \\
\hline \multicolumn{13}{|l|}{ Education } \\
\hline$<$ High School & 1051 & 280 & $40.2(35.5,45.1)$ & 207 & $38.0(32.8,43.4)$ & 235 & $36.2(31.5,41.3)$ & 181 & $34.6(29.4,40.1)$ & 127 & $42.0(35.2,49.0)$ & -0.06 \\
\hline HS/GED & 2722 & 788 & $42.0(39.0,45.0)$ & 493 & $40.6(37.1,44.1)$ & 628 & $39.5(36.5,42.6)$ & 440 & $40.1(36.8,43.6)$ & 324 & $40.4(36.3,44.5)$ & -0.90 \\
\hline Some Post HS & 3371 & 913 & $51.8(48.8,54.7)$ & 559 & $47.8(44.4,51.3)$ & 820 & $48.9(45.9,52.0)$ & 602 & $50.3(47.0,53.6)$ & 397 & $46.3(42.2,50.4)$ & -1.72 \\
\hline College Grad & 4379 & 1185 & $46.5(44.0,48.9)$ & 696 & $48.3(45.3,51.5)$ & 1066 & $47.0(44.5,49.5)$ & 766 & $46.0(43.2,48.8)$ & 558 & $45.8(42.7,49.1)$ & -0.79 \\
\hline $\begin{array}{c}\text { Do not } \\
\text { know/Refused }\end{array}$ & 20 & 8 & $44.9(17.4,75.8)$ & 6 & $58.7(29.5,82.8)$ & 4 & $40.2(14.6,72.6)$ & 1 & $3.1(0.4,21.7)$ & 2 & $8.8(1.4,39.2)$ & - \\
\hline
\end{tabular}


Table 2. Cont.

\begin{tabular}{|c|c|c|c|c|c|c|c|c|c|c|c|c|}
\hline & Total & & 2011 & & 2012 & & 2013 & & 2014 & & 2015 & \\
\hline & Unwt. N & Unwt. $\mathbf{N}$ & Wt. $\%(95 \% \mathrm{CI})$ & Unwt. $\mathbf{N}$ & Wt. \% $(95 \% \mathrm{CI})$ & Unwt. $\mathbf{N}$ & Wt. $\%(95 \% \mathrm{CI})$ & Unwt. $\mathbf{N}$ & Wt. \% $(95 \% \mathrm{CI})$ & Unwt. $\mathbf{N}$ & Wt. \% $(95 \%$ CI $)$ & APC * \\
\hline \multicolumn{13}{|l|}{$\begin{array}{l}\text { Annual income } \\
(\text { USD (\$)) }\end{array}$} \\
\hline$<15,000$ & 1476 & 394 & $52.6(47.5,57.6)$ & 308 & $51.1(45.6,56.6)$ & 353 & $49.9(45.3,54.5)$ & 249 & $47.3(41.4,53.2)$ & 150 & $48.7(41.4,56.0)$ & $-2.29 *$ \\
\hline $15,000-<25,000$ & 1995 & 570 & $49.7(45.7-53.6)$ & 350 & $46.8(42.3-51.4)$ & 475 & $46.7(42.7-50.7)$ & 340 & $42.6(38.2,47.1)$ & 231 & $46.7(41.2,52.3)$ & -2.16 \\
\hline $25,000-<35,000$ & 1171 & 335 & $45.3(40.5-50.2)$ & 201 & $45.0(39.2-50.9)$ & 267 & $46.6(41.5-51.8)$ & 201 & $46.3(40.7,52.0)$ & 111 & $40.4(33.7,47.5)$ & -1.98 \\
\hline $35,000-<50,000$ & 1332 & 377 & $46.1(41.6,50.6)$ & 206 & $42.3(37.1,47.7)$ & 337 & $47.4(42.7,52.1)$ & 232 & $47.2(42.0,52.5)$ & 160 & $49.4(43.1,55.8)$ & 2.51 \\
\hline $50,000+$ & 4461 & 1197 & $45.0(42.5,47.4)$ & 710 & $43.5(40.5,46.5)$ & 1034 & $42.5(40.0,45.1)$ & 771 & $43.7(40.9,46.5)$ & 562 & $43.9(40.6,47.2)$ & -0.45 \\
\hline $\begin{array}{c}\text { Do not } \\
\text { know/Refused }\end{array}$ & 1108 & 301 & $34.9(30.3,39.8)$ & 186 & $36.1(30.9,41.7)$ & 287 & $32.1(27.8,36.7)$ & 197 & $36.8(31.8,42.1)$ & 194 & $35.1(29.8,40.7)$ & - \\
\hline \multicolumn{13}{|l|}{ Marital status } \\
\hline Married & 5534 & 1586 & $41.1(39.2,43.1)$ & 890 & $36.6(34.3,39.0)$ & 1218 & $38.4(36.3,40.5)$ & 919 & $39.1(36.8,41.5)$ & 654 & $37.6(34.9,40.4)$ & -1.11 \\
\hline Divorced & 2116 & 574 & $51.6(47.6,55.6)$ & 346 & $55.0(50.1,59.8)$ & 540 & $56.4(51.9,60.8)$ & 363 & $53.1(48.2,57.8)$ & 257 & $54.2(48.6,59.7)$ & 0.63 \\
\hline Widowed & 704 & 204 & $21.6(18.3,25.2)$ & 127 & $21.7(17.4,26.7)$ & 166 & $20.6(17.2,24.6)$ & 151 & $22.8(18.4,27.9)$ & 98 & $29.7(23.8,36.4)$ & 7.10 \\
\hline Separated & 512 & 143 & $68.5(59.4,76.4)$ & 87 & $69.5(58.7,78.6)$ & 140 & $68.9(60.0,76.6)$ & 88 & $66.5(55.4,76.0)$ & 56 & $62.5(49.1,74.1)$ & -2.25 \\
\hline Never married & 2255 & 553 & $53.7(49.5,57.9)$ & 440 & $54.8(50.3,59.1)$ & 585 & $50.2(46.3,54.1)$ & 400 & $50.4(46.0,54.8)$ & 290 & $53.3(47.9,58.5)$ & -0.98 \\
\hline $\begin{array}{l}\text { A member of an } \\
\text { unmarried couple }\end{array}$ & 366 & 100 & $53.6(43.9,63.1)$ & 64 & $61.5(48.9,72.6)$ & 90 & $50.3(41.4,59.1)$ & 59 & $50.2(38.8,61.5)$ & 43 & $44.2(33.0,55.9)$ & -5.72 \\
\hline Refused & 56 & 14 & $39.5(18.4,65.4)$ & 7 & $35.6(12.0,69.1)$ & 14 & $42.5(23.5,64.0)$ & 10 & $53.4(28.6,76.7)$ & 10 & $29.4(13.3,53.1)$ & - \\
\hline \multicolumn{13}{|l|}{$\begin{array}{l}\text { Health care } \\
\text { coverage }\end{array}$} \\
\hline Yes & 9216 & 2541 & $43.0(41.3,44.7)$ & 1524 & $41.2(39.3,43.2)$ & 2157 & $40.6(38.9,42.3)$ & 1662 & $42.2(40.4,44.1)$ & 1215 & $42.4(40.1,44.6)$ & -0.04 \\
\hline No & 2303 & 629 & $53.6(49.6,57.6)$ & 435 & $54.8(50.2,59.3)$ & 584 & $53.9(49.9,57.8)$ & 324 & $49.5(44.6,54.4)$ & 187 & $51.2(44.6,57.7)$ & -1.91 \\
\hline $\begin{array}{c}\text { Do not } \\
\text { know/Refused }\end{array}$ & 24 & 4 & $12.6(2.3,43.3)$ & 2 & $23.4(2.6,77.4)$ & 12 & $51.0(20.4,82.2)$ & 4 & $37.0(9.6,76.4)$ & 6 & $43.9(8.0,71.6)$ & - \\
\hline $\begin{array}{l}\text { HIV high risk } \\
\text { situations }\end{array}$ & $N=5114$ & $n=3164$ & & $n=1950$ & & & & & & & & \\
\hline Yes & 211 & 130 & $74.0(63.8,82.1)$ & 81 & $69.4(58.1,78.7)$ & $\mathrm{N} / \mathrm{A}$ & $\mathrm{N} / \mathrm{A}$ & N/A & N/A & N/A & $\mathrm{N} / \mathrm{A}$ & - \\
\hline No & 4884 & 3020 & $44.2(42.6,45.8)$ & 1864 & $43.2(41.3,45.1)$ & N/A & N/A & $\mathrm{N} / \mathrm{A}$ & $\mathrm{N} / \mathrm{A}$ & N/A & N/A & - \\
\hline $\begin{array}{c}\text { Do not } \\
\text { know/Refused }\end{array}$ & 19 & 14 & $75.3(41.8,90.0)$ & 5 & $25.0(6.0,49.4)$ & $N / A$ & N/A & $N / A$ & N/A & $\mathrm{N} / \mathrm{A}$ & $\mathrm{N} / \mathrm{A}$ & - \\
\hline Overall USA & 608,484 & 132,471 & $35.9(35.6,36.2)$ & 128,927 & $35.3(35.0,35.5)$ & 130,922 & $35.9(35.6,36.2)$ & 115,866 & $34.4(34.1,34.7)$ & 113,779 & $38.0(37.7,38.3)$ & 0.88 \\
\hline
\end{tabular}

Acronyms: Unwt. N—unweighted counts, Wt. \%—weighted population estimates, CI—confidence interval, APC—Annual Percent Change, NH—non-Hispanic, GED—General Educational Development, N/A—data not available. Note: $p$-values were significant for the association between all the socio-demographic categories and having ever been tested for HIV. * APC was not significantly different from zero at alpha $=0.05$ for all the variables, except annual income $<\$ 15,000$. (-) APC was not calculated for respondents who do not know or refused to respond to questions asked, and for HIV high risk situations. USD-United States Dollar. 


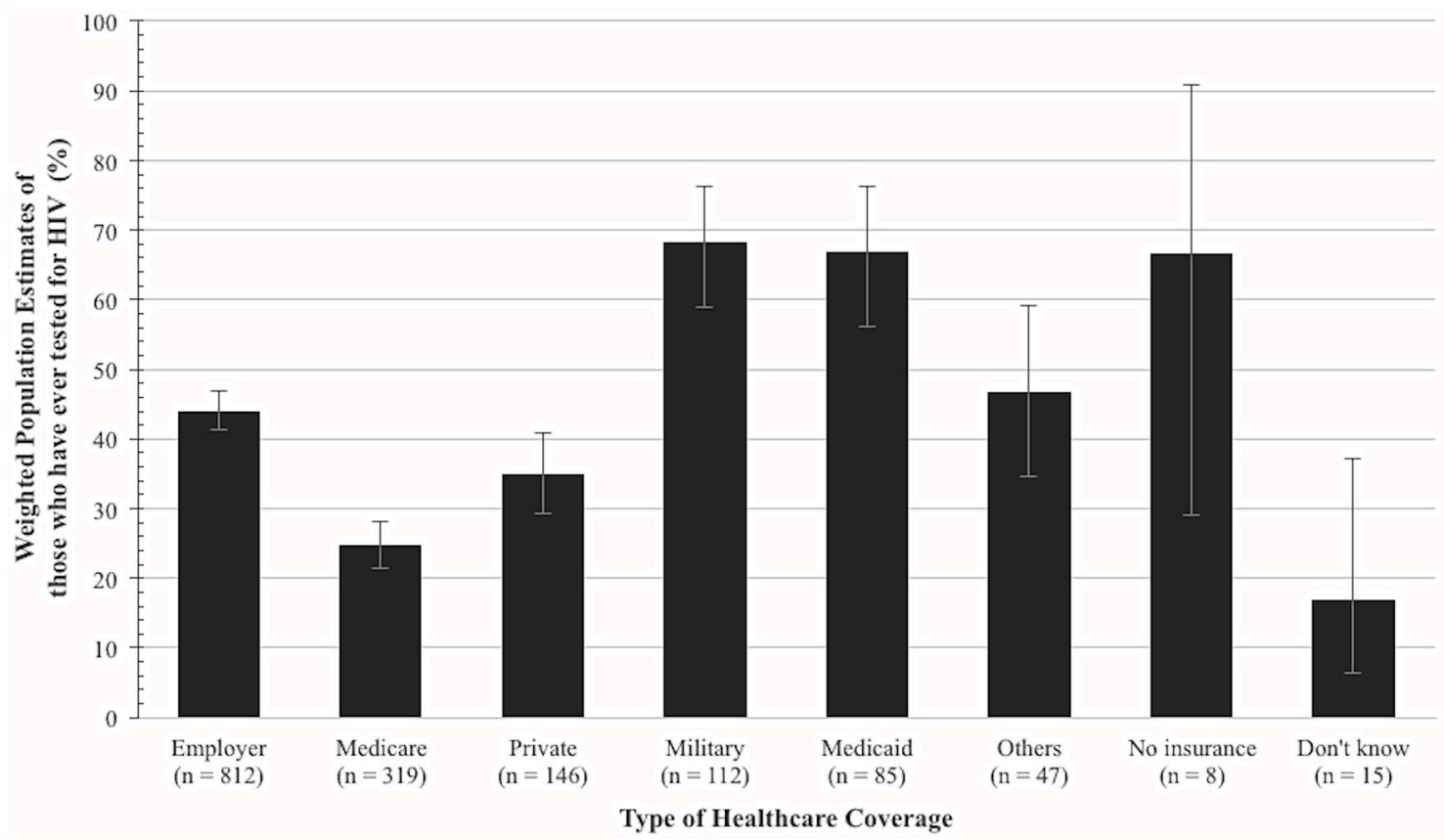

Figure 1. Weighted percentages of adults who have ever tested for HIV in Georgia based on the type of healthcare coverage: BRFSS 2014 data. Note: Error bars indicate 95\% confidence intervals for each estimate.

\subsection{Socio-Demographic Determinants of HIV Testing in GA}

In Table 3 are the results of logistic regression analyses for the association between socio-demographic factors and the dependent variable, having ever been tested for HIV, after adjusting for all the variables in the model. Excluded from the model is the variable HIV high-risk situations, because data were available only for 2011 and 2012. Except for healthcare coverage, all the variables entered had a significant effect on the model. Females (OR $=1.13(95 \% \mathrm{CI}=1.04,1.23) ; p=0.004), \mathrm{NH}$ black/African American respondents $(\mathrm{OR}=2.82(95 \% \mathrm{CI}=2.54,3.12) ; p<0.001)$, and respondents of other ethnic groups combined ( $\mathrm{OR}=0.97(95 \% \mathrm{CI}=0.85,1.12) ; p=0.70)$ were more likely to have been tested than males, and NH white respondents, respectively. People who were younger than 55 (18-54 years) were more likely than older people to have tested for HIV (OR $=2.58$ (95\% CI $=2.31$, 2.89); $p<0.001)$. The likelihood of being tested for HIV was also associated with levels of education greater than high school $(\mathrm{OR}=1.46(95 \% \mathrm{CI}=1.31,1.63) ; p<0.001)$, being single $(\mathrm{OR}=1.22(95 \%$ $\mathrm{CI}=1.11,1.34) ; p<0.001)$, and earning annual income of less than $\$ 50,000(\mathrm{OR}=1.18(95 \% \mathrm{CI}=1.04$, $1.34) ; p=0.01$ for $\langle \$ 25,000$ and $\mathrm{OR}=1.14(95 \% \mathrm{CI}=1.01,1.28) ; p=0.03$ for $\$ 25,000-\$ 50,000$ annual income), compared to less than high school education, being in a couple relationship, and earning more than $\$ 50,000$ annually, respectively. Having healthcare coverage was not significantly associated with HIV testing. 
Table 3. Logistic regression analysis of socio-demographic factors associated with having ever been tested for HIV in Georgia: BRFSS 2011-2015.

\begin{tabular}{|c|c|c|c|}
\hline Variable & Odds Ratio & $95 \%$ CI & $p$-Value \\
\hline $\begin{array}{l}\text { Gender } \\
\text { Female } \\
\text { Male }\end{array}$ & $\begin{array}{c}1.134 \\
\text { Reference }\end{array}$ & $1.041,1.234$ & 0.004 \\
\hline $\begin{array}{c}\text { Age (years) } \\
18-34 \\
35-54 \\
\geq 55\end{array}$ & $\begin{array}{c}2.583 \\
2.578 \\
\text { Reference }\end{array}$ & $\begin{array}{l}2.306,2.894 \\
2.344,2.836\end{array}$ & $\begin{array}{l}<0.001 \\
<0.001\end{array}$ \\
\hline $\begin{array}{c}\text { Race } \\
\text { Black, NH } \\
\text { Others } \\
\text { White, NH }\end{array}$ & $\begin{array}{c}2.815 \\
0.973 \\
\text { Reference }\end{array}$ & $\begin{array}{l}2.538,3.122 \\
0.845,1.120\end{array}$ & $\begin{array}{c}<0.001 \\
0.701\end{array}$ \\
\hline $\begin{array}{c}\text { Education } \\
\text { College graduate } \\
\text { Some post high school } \\
\leq \mathrm{HS}\end{array}$ & $\begin{array}{c}1.463 \\
1.462 \\
\text { Reference }\end{array}$ & $\begin{array}{l}1.313,1.631 \\
1.311,1.630\end{array}$ & $\begin{array}{l}<0.001 \\
<0.001\end{array}$ \\
\hline $\begin{array}{c}\text { Income } \\
<25,000 \\
25,000-50,000 \\
>50,000\end{array}$ & $\begin{array}{c}1.178 \\
1.138 \\
\text { Reference }\end{array}$ & $\begin{array}{l}1.035,1.342 \\
1.014,1.277\end{array}$ & $\begin{array}{l}0.013 \\
0.028\end{array}$ \\
\hline $\begin{array}{l}\text { Marital status } \\
\text { Single } \\
\text { Couple }\end{array}$ & $\begin{array}{c}1.216 \\
\text { Reference }\end{array}$ & $1.106,1.337$ & $<0.001$ \\
\hline $\begin{array}{c}\text { Healthcare coverage } \\
\text { No } \\
\text { Yes }\end{array}$ & $\begin{array}{c}1.046 \\
\text { Reference }\end{array}$ & $0.927,1.179$ & 0.467 \\
\hline
\end{tabular}

\section{Discussion}

BRFSS data from 2011 to 2015 (the years of the most current data) were analyzed to examine the temporal trends and socio-demographic factors associated with HIV testing among adults in GA. Overall in GA, there was a slight decrease in the percentages of respondents who had ever been tested for HIV, from 45.6\% in 2011 to 43.7\% in 2015, with a non-significant APC. During this time, the annual percentages of those tested were higher for GA compared to the national rates (35.9\% in $2011,38.0 \%$ in 2015). The factors associated with HIV testing included being female, black/African American, single, younger than 55 years, having greater than high school education, and earning $\$ 50,000$ or less annually.

The results of the current study show that the HIV testing trends were stable between 2011 and 2015, however, less than half of the adults living in GA had been tested for HIV. Results of earlier studies conducted nationally and in other parts of the U.S. $[27,28]$ show that the percentages of adults who had ever tested for HIV increased significantly between 2000 and 2010 (36.6\% in 2000, 45.0\% in 2010, $p<0.0001$ [27]. A study that analyzed data from the Southeastern Pennsylvania Household Health Survey between 2002 and 2010 to evaluate HIV testing over time, reported that testing trends increased among all demographic groups, but existing differences in testing before 2006 persisted after that year as follows: younger patients, racial/ethnic minorities, and patients on Medicaid were all more likely to get tested than their counterparts [28].

Barriers to HIV testing include HIV-related stigma, sexuality, religion, race, and class, emphasizing responsibility, testing concerns, and media influences $[29,30]$. The percentages of respondents who had been tested for HIV were highly associated with the presence of HIV risk factors and with self-reported current risks of contracting HIV. Racial minorities, younger persons, especially young black/African American (men having sex with men (MSM)), have the highest risk and prevalence of HIV / AIDS [28]. 
Gay, bisexual, and other men who have sex with men accounted for an estimated $2 \%$ of the total population, and 55.0\% of people living with HIV in the United States in 2013 [31]. Georgia is among the states with the highest population of MSM and African Americans [7,32], and this may account for the higher rate of HIV testers in GA, compared to the national rate. HIV risk is associated with low socio-economic status among heterosexual populations [33] and the current study shows that lower income earners and those without healthcare coverage were more likely to test for HIV. However, this does not explain why the participants that attained educational levels greater than high school tested more than those with high school or lesser education. A possible explanation may be that a great proportion of the study participants were educated beyond high school.

A similar study by Handel et al. (2016), analyzed the BRFSS data for 2011-2013, and reported that a national average of $33 \%$ of young adults (18-24 years) had tested for HIV [34]; the average for the same age group in GA was approximately $40.0 \%$ as reported by the current study. The Handel study also showed that a significant decrease in the prevalence of HIV testing was detected overall from $42.4 \%$ in 2011 to $39.5 \%$ in 2013 among young adult females nationally, with significant racial/ethnic differences in the rates of decline $(9.0 \%$ decrease among young adult black females, and $3.3 \%$ decrease among young adult white females).

A disadvantage of low HIV testing among persons who are perceived as low-risk is the missing of opportunities to diagnose HIV-infected persons and linking them to care. Reasons for fewer people with healthcare coverage not being tested may be because (a) routine HIV testing is not offered in the places where most people get their health care and (b) awareness of CDC's 2006 recommendations for HIV screening has been low among primary care providers [35,36]. Release of the United States Preventive Services Task Force recommendations for HIV testing in 2013, and the provision of the Affordable Care Act that both HIV screening and targeted risk-based testing are now covered without cost-sharing as part of the essential benefits package, may boost future HIV testing rates [36].

A strength of the present report is the utilization of the most currently available BRFSS data. It is also among the few studies that have examined the trends of HIV testing with the APCs, and the associated socio-demographic factors of HIV testing and is the only report solely for the state of GA. There are some limitations. The BRFSS data are self-reported by respondents and are subject to recall bias. The survey is based on non-institutionalized populations and excludes persons with the same risk of exposure who are residing elsewhere, such as nursing homes or long-term-care facilities. Since data are collected by telephone, individuals who live in households without a residential telephone or cell phone are not included. Further, the sampling frame of the BRFSS is the entire state; therefore, some rural areas might be represented by relatively few interviews. Because of these limitations, the results might be either underestimated or overestimated. Despite these limitations, data from the BRFSS are reliable and generally valid because the content of the survey questions, questionnaire design, data collection, procedures, interviewing techniques, and data processing have been developed to improve data quality [37].

\section{Conclusions}

Between 2011 and 2015, the percentage of adults in GA who have ever been tested for HIV has remained stable, with less than $50 \%$ now reporting to have been tested. In GA, increasing access to and utilization of HIV testing should be a public health priority, and more programs that will increase awareness to recommendations for testing among healthcare providers are warranted.

Acknowledgments: This work was funded by the National Cancer Institute (1R01CA166785) and the National Institute on Minority Health and Health Disparities (1P20MD006881).

Author Contributions: Benjamin Ansa contributed to the conception, design, statistical analysis, writing, and submission of the manuscript; Yunmi Chung and Sashia White contributed to the statistical analysis and editing of the manuscript; and Selina A. Smith reviewed and edited the manuscript. All authors read and approved the final manuscript.

Conflicts of Interest: The authors declare no conflict of interest. 


\section{References}

1. Steward, W.T.; Remien, R.H.; Higgins, J.A.; Dubrow, R.; Pinkerton, S.D.; Sikkema, K.J.; Truong, H.-H.M.; Johnson, M.O.; Hirsch, J.; Brooks, R.A. Behavior change following diagnosis with acute/early HIV infection-A move to serosorting with other HIV-infected individuals. The NIMH Multisite Acute HIV Infection Study: III. AIDS Behav. 2009, 13, 1054-1060. [CrossRef] [PubMed]

2. Marks, G.; Crepaz, N.; Janssen, R.S. Estimating sexual transmission of HIV from persons aware and unaware that they are infected with the virus in the USA. Aids 2006, 20, 1447-1450. [CrossRef] [PubMed]

3. Higginbotham, S.; Holmes, R.; Stone, H.; Beil, J.; Datu, G.B.; Costa, S.; Paul, S. Adoption of protective behaviors among persons with recent HIV infection and diagnosis-Alabama, New Jersey, and Tennessee, 1997-1998 (Reprinted from MMWR, 2000 49, 512-515). JAMA 2000, 284, 171-173.

4. Centers for Disease Control and Prevention. HIV/AIDS. HIV Testing. Available online: http://www.cdc. gov/hiv/testing/index.html (accessed on 25 August 2016).

5. Schwarcz, S.K.; Hsu, L.; Chin, C.-S.J.; Richards, T.A.; Frank, H.; Wenzel, C.; Dilley, J. Do people who develop AIDS within 12 months of HIV diagnosis delay HIV testing? Public Health Rep. 2011, 126, 552-559. [PubMed]

6. Centers for Disease Control and Prevention. HIV/AIDS. HIV in the United States: At a Glance. June 2016. Available online: http://www.cdc.gov/hiv/statistics/overview/ataglance.html (accessed on 25 August 2016).

7. United States Census Bureau. Quick Facts. Georgia. Available online: http://www.census.gov/quickfacts/ table/PST045215/13 (accessed on 25 August 2016).

8. Centers for Disease Control and Prevention, NCHHSTP Newsroom. Lifetime Risk of HIV Diagnosis. Available online: http://www.cdc.gov/nchhstp/newsroom/2016/croi-press-release-risk.html (accessed on 25 August 2016).

9. Georgia Department of Public Health. HIV Surveillance Fact Sheet 2014. Available online: http://dph. georgia.gov/georgias-hivaids-epidemiology-surveillance-section (accessed on 25 August 2016).

10. AIDSVu. Georgia Highlights. Available online: http://aidsvu.org/state/georgia/ (accessed on 25 August 2016).

11. Branson, B.M.; Handsfield, H.H.; Lampe, M.A.; Janssen, R.S.; Taylor, A.W.; Lyss, S.B.; Clark, J.E. Revised recommendations for HIV testing of adults, adolescents, and pregnant women in health-care settings. J. Natl. Med. Assoc. 2008, 100, 131-147.

12. Millett, G.A.; Crowley, J.S.; Koh, H.; Valdiserri, R.O.; Frieden, T.; Dieffenbach, C.W.; Fenton, K.A.; Benjamin, R.; Whitescarver, J.; Mermin, J. A way forward: The National HIV/AIDS Strategy and reducing HIV incidence in the United States. JAIDS 2010, 55, S144-S147. [CrossRef] [PubMed]

13. Centers for Disease Control and Prevention, Behavioral Risk Factor Surveillance System (BRFSS). About BRFSS. Available online: http:/ /www.cdc.gov/brfss/about/index.htm (accessed on 25 August 2016).

14. Georgia Department of Public Health, Georgia Behavioral Risk Factor Surveillance System (BRFSS). Available online: https://dph.georgia.gov/georgia-behavioral-risk-factor-surveillance-system-brfss (accessed on 25 August 2016).

15. Centers for Disease Control and Prevention, Behavioral Risk Factor Surveillance System. Overview: BRFSS 2015. July 2016. Available online: http://www.cdc.gov/brfss/annual_data/2015/pdf/overview_2015.pdf (accessed on 25 October 2016).

16. American Association for Public Opinion Research. Standard Definitions. Available online: https://www. aapor.org/AAPOR_Main/media/publications/Standard-Definitions2015_8theditionwithchanges_April2015_ logo.pdf (accessed on 25 October 2016).

17. Centers for Disease Control and Prevention. Behavioral Risk Factor Surveillance System. 2011 Summary Data Quality Report (Version \#5—Revised: 2 April 2013). Available online: http:/ /www.cdc.gov/brfss / annual_data/2011/pdf/2011_summary_data_quality_report.pdf (accessed on 25 October 2016).

18. Centers for Disease Control and Prevention. Behavioral Risk Factor Surveillance System. 2012 Summary Data Quality Report (3 July 2013). Available online: http:/ /www.cdc.gov/brfss/annual_data/2012/pdf/ summarydataqualityreport2012_20130712.pdf (accessed on 25 October 2016).

19. Centers for Disease Control and Prevention. Behavioral Risk Factor Surveillance System. 2013 Summary Data Quality Report. (15 August 2014). Available online: http://www.cdc.gov/brfss/annual_data/2013/ pdf/2013_dqr.pdf (accessed on 25 October 2016). 
20. Centers for Disease Control and Prevention. Behavioral Risk Factor Surveillance System. 2014 Summary Data Quality Report. (29 July 2015). Available online: http:/ /www.cdc.gov/brfss/annual_data/2014/pdf/ 2014_dqr.pdf (accessed on 25 October 2016).

21. Centers for Disease Control and Prevention. Behavioral Risk Factor Surveillance System. 2015 Summary Data Quality Report. (29 July 2015). Available online: http:/ /www.cdc.gov/brfss/annual_data/2015/pdf/ 2015-sdqr.pdf (accessed on 25 October 2016).

22. Centers for Disease Control and Prevention. Behavioral Risk Factor Surveillance System. Weighting BRFSS Data BRFSS 2014. Available online: https://www.cdc.gov/brfss/annual_data/2014/pdf/weighting-data. pdf (accessed on 25 October 2016).

23. Joinpoint Regression Program, Version 4.3.1.0-April 2016; Statistical Methodology and Applications Branch; Surveillance Research Program; National Cancer Institute. Available online: https://surveillance.cancer. gov/help/joinpoint/tech-help/citation (accessed on 25 October 2016).

24. Kim, H.-J.; Fay, M.P.; Feuer, E.J.; Midthune, D.N. Permutation tests for joinpoint regression with applications to cancer rates. Stat. Med. 2000, 19, 335-351. [CrossRef]

25. IBM Corp. IBM SPSS Statistics for Windows; Version 24.0; IBM Corp: Armonk, NY, USA, 2016.

26. Bernstein, A.B.; Sweeney, M.H.; Centers for Disease Control and Prevention. Public health surveillance data: Legal, policy, ethical, regulatory, and practical issues. MMWR Suppl. 2012, 61, 30-34. [PubMed]

27. Centers for Disease Control and Prevention. HIV Testing Trends in the United States, 2000-2011; U.S. Department of Health and Human Services; Centers for Disease Control and Prevention: Atlanta, GA, USA, 2013.

28. Momplaisir, F.; Yehia, B.R.; Harhay, M.O.; Fetzer, B.; Brady, K.A.; Long, J.A. HIV testing trends: Southeastern Pennsylvania, 2002-2010. AIDS Patient Care STDs 2014, 28, 303-310. [CrossRef] [PubMed]

29. Nanín, J.; Osubu, T.; Walker, J.N.; Powell, B.; Powell, D.; Parsons, J. "HIV is still real”: Perceptions of HIV testing and HIV prevention among black men who have sex with men in New York City. Am. J. Men's Health 2009, 3, 150-164. [CrossRef] [PubMed]

30. Nunn, A.; Eng, W.; Cornwall, A.; Beckwith, C.; Dickman, S.; Flanigan, T.; Kwakwa, H. African American patient experiences with a rapid HIV testing program in an urban public clinic. J. Natl. Med. Assoc. 2012, 104, 5. [CrossRef]

31. Centers for Disease Control and Prevention. HIV among Gay and Bisexual Men. Available online: https:// www.cdc.gov/hiv/group/msm/ (accessed on 25 October 2016).

32. Grey, J.A.; Bernstein, K.T.; Sullivan, P.S.; Purcell, D.W.; Chesson, H.W.; Gift, T.L.; Rosenberg, E.S. Estimating the Population Sizes of Men Who Have Sex With Men in US States and Counties Using Data From the American Community Survey. JMIR Public Health Surveill. 2016, 2, e14. [CrossRef] [PubMed]

33. Centers for Disease Control and Prevention. Characteristics associated with HIV infection among heterosexuals in urban areas with high AIDS prevalence-24 cities, United States, 2006-2007. Morb. Mortal. Wkly. Rep. 2011, 60, 1045.

34. Van Handel, M.; Kann, L.; Olsen, E.O.M.; Dietz, P. HIV testing among us high school students and young adults. Pediatrics 2016, 137, e20152700. [CrossRef] [PubMed]

35. Dietz, P.M.; van Handel, M.; Wang, H.; Peters, P.J.; Zhang, J.; Viall, A.; Branson, B.M. HIV Testing among Outpatients with Medicaid and Commercial Insurance. PLoS ONE 2015, 10, e0144965. [CrossRef] [PubMed]

36. Al Mohajer, M.; Lyons, M.; King, E.; Pratt, J.; Fichtenbaum, C.J. Internal medicine and emergency medicine physicians lack accurate knowledge of current CDC HIV testing recommendations and infrequently offer HIV testing. J. Int. Assoc. Phys. AIDS Care 2012, 11, 101-108. [CrossRef] [PubMed]

37. Barrett-Connor, E.; Ayanian, J.Z.; Brown, E.R.; Coultas, D.B.; Francis, C.K.; Goldberg, R.J.; Gostin, L.O.; Kottke, T.E.; Lee, E.T.; Mannino, D.M. A Nationwide Framework for Surveillance of Cardiovascular and Chronic Lung Diseases; National Academies Press (U.S.): Washington, DC, USA, 2011.

(C) 2016 by the authors; licensee MDPI, Basel, Switzerland. This article is an open access article distributed under the terms and conditions of the Creative Commons Attribution (CC-BY) license (http://creativecommons.org/licenses/by/4.0/). 